\title{
Inhibition of mitoNEET attenuates LPS-induced inflammation and oxidative stress
}

\author{
Seunghee Lee $\mathbb{D}^{1}$, Byeong Geun Seok $\mathbb{D}^{1}$, Seon-Jin Lee $\mathbb{D}^{2}$ and Su Wol Chung $\mathbb{D}^{1 \times}$ \\ (c) The Author(s) 2022
}

\begin{abstract}
MitoNEET (mitochondrial protein containing Asn-Glu-Glu-Thr (NEET) sequence) is a $2 \mathrm{Fe}-2 \mathrm{~S}$ cluster-containing integral membrane protein that resides in the mitochondrial outer membrane and participates in a redox-sensitive signaling and Fe-S cluster transfer. Thus, mitoNEET is a key regulator of mitochondrial oxidative capacity and iron homeostasis. Moreover, mitochondrial dysfunction and oxidative stress play critical roles in inflammatory diseases such as sepsis. Increased iron levels mediated by mitochondrial dysfunction lead to oxidative damage and generation of reactive oxygen species (ROS). Increasing evidence suggests that targeting mitoNEET to reverse mitochondrial dysfunction deserves further investigation. However, the role of mitoNEET in inflammatory diseases is unknown. Here, we investigated the mechanism of action and function of mitoNEET during lipopolysaccharide (LPS)induced inflammatory responses in vitro and in vivo. Levels of mitoNEET protein increased during microbial or LPS-induced sepsis. Pharmacological inhibition of mitoNEET using mitoNEET ligand-1 (NL-1) decreased the levels of pro-inflammatory cytokines such as IL-1 $\beta$, IL-6, and TNF- $\alpha$ in animal models of sepsis, as well as LPS-induced inflammatory responses by macrophages in vitro. Inhibition of mitoNEET using NL-1 or mitoNEET shRNA abrogated LPS-induced ROS formation and mitochondrial dysfunction. Furthermore, mitochondrial iron accumulation led to generation of LPS-induced ROS, a process blocked by NL-1 or shRNA. Taken together, these data suggest that mitoNEET could be a key therapeutic molecule that targets mitochondrial dysfunction during inflammatory diseases and sepsis.
\end{abstract}

Cell Death and Disease (2022)13:127; https://doi.org/10.1038/s41419-022-04586-2

\section{INTRODUCTION}

Inflammation is critical for healing, but uncontrolled and dysregulated inflammation can increase the risk of developing various diseases [1]. Sepsis, caused mainly by bacterial infection, is a highly inflammatory disorder that, in severe cases, can cause organ dysfunction and death [2]. The link between sepsisassociated organ failure and mitochondrial dysfunction is increasing interest to researchers [3]. Sepsis-induced mitochondrial dysfunction mediates hyperinflammation through cellular metabolic disorders, insufficient energy production, and oxidative stress; as such, it plays a key role in the development of sepsisrelated multiorgan failure [4-6]. Mitochondria, dynamic organelles that serve as the power house of the cell, are a major source of reactive oxygen species (ROS); they are also the site where iron is transformed into its bioactive form [7-10]. Increased mitochondrial iron accumulation due to pro-inflammatory signaling promotes oxidative damage by catalyzing generation of ROS and causing mitochondrial dysfunction $[9,11]$. These processes develop into a vicious inflammatory cycle [12]. Therefore, the mitochondrial iron level must be strictly regulated to avoid ironmediated damage and maintain mitochondrial function. Several studies demonstrate that targeting mitochondrial iron accumulation using iron chelators has the potential to improve the prognosis of sepsis $[8,13,14]$.
The mitochondrial protein mitoNEET containing Asn-Glu-Glu-Thr (NEET) sequence (also referred to as CDGSH (C-X-C-X2-(S/T)-X3-P-X-C-D-G-(S/A/T)-H) iron sulfur domain 1 $(C I S D 1))$ is a $2 \mathrm{Fe}-2 \mathrm{~S}$ cluster-containing, redox-sensitive protein that resides on the outer mitochondrial membrane; as such, it is a powerful regulator of mitochondrial iron content [15-17]. Only when the mitoNEET [2Fe-2S] clusters are oxidized do they transfer [2Fe-2S] clusters to apo-proteins and electrons from $\mathrm{FMNH} 2$ (reduced 1,5-dihydro form of flavin mononucleotide) to oxygen or ubiquinone in mitochondria [18-20]. Therefore, mitoNEET exerts marked effects on cellular and systemic metabolic homeostasis by acting as a powerful regulator of mitochondrial iron content. Early studies showed that mitoNEET plays a key role in regulating cellular energy use, lipid metabolism, and cancer cell proliferation and tumor formation [21-23]. Recent studies on the effects of redox regulation by mitoNEET demonstrate that mice overexpressing mitoNEET exhibit reduced ROS generation by mitochondria; however, oxidative phosphorylation and electron transport are significantly upregulated in the absence of mitoNEET $[6,24]$. This is associated with generation of ROS by mitochondria, along with mitochondrial dysfunction [25]. Thus, mitoNEET is involved in a variety of human pathologies, including cystic fibrosis, diabetes, muscle atrophy, and neurodegeneration [25-27].

\footnotetext{
${ }^{1}$ School of Biological Sciences, College of Natural Sciences, University of Ulsan, 93 Daehak-ro, Nam-gu, Ulsan 44610, South Korea. ${ }^{2}$ Genome Structure Research Center, Korea Research Institute of Bioscience and Biotechnology, Yuseong-gu, Daejeon 305-806, South Korea. ${ }^{\circledR}$ email: swchung@ulsan.ac.kr Edited by Professor Hans-Uwe Simon
} 


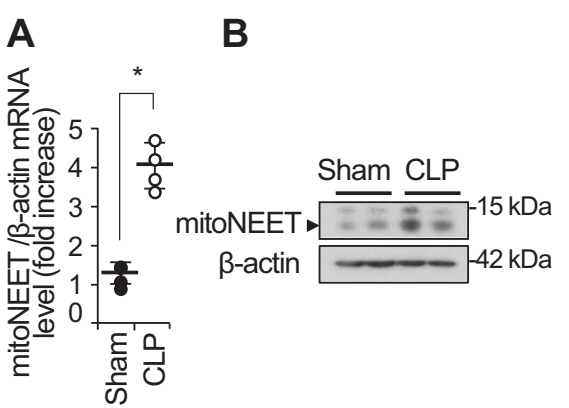

E

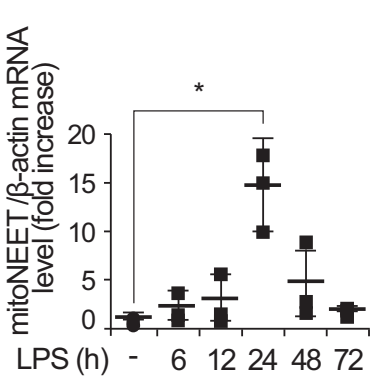

G

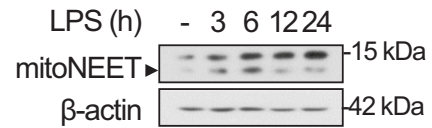

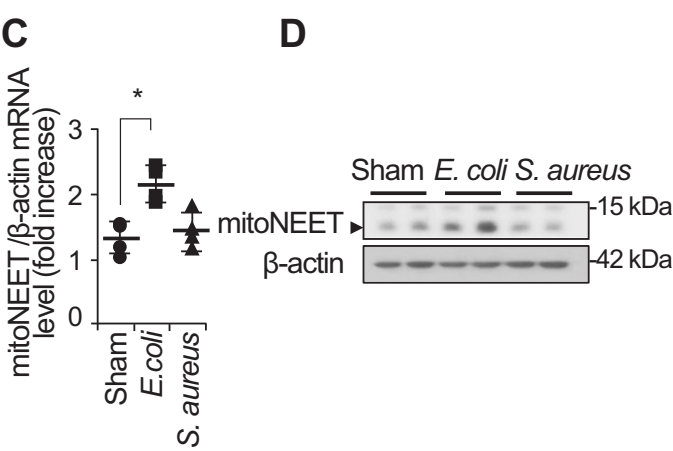

$\mathbf{F}$

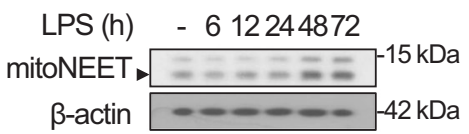

$\mathbf{H}$

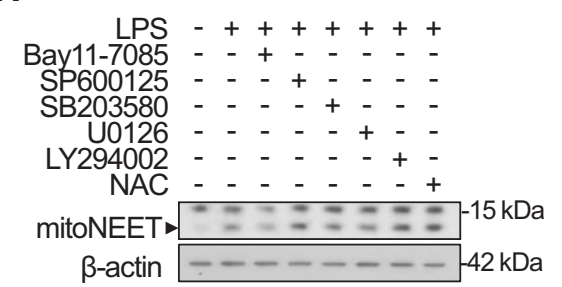

Fig. 1 Expression of mitoNEET mRNA and protein increases during microbial sepsis. Total RNA and protein were extracted from the spleen $48 \mathrm{~h}$ after sham or CLP surgery, and fibrin clot-induced microbial sepsis was triggered by $E$. coli or S. aureus bacteria ( $\left.1 \times 10^{8} \mathrm{CFU}\right)$. Expression of mitoNEET mRNA $(\mathbf{A}, \mathbf{C})$ and protein $(\mathbf{B}, \mathbf{D})$ levels was assessed by quantitative real-time RT-PCR or western blotting. ${ }^{*} P<0.05$ for sham vs. CLP or fibrin clot-induced microbial sepsis. C57BL/6 mice were injected with LPS $(20 \mathrm{mg} / \mathrm{kg})$ or vehicle, and total RNA and protein were extracted from the spleen $6,12,24,48$, and $72 \mathrm{~h}$ after administration of vehicle or LPS (100 ng/mL). Total protein was extracted from BMDMs $3,6,12$, and $24 \mathrm{~h}$ after administration of vehicle or LPS $(100 \mathrm{ng} / \mathrm{mL})$. Expression of mitoNEET mRNA $(\mathbf{E})$ and protein $(\mathbf{F}, \mathbf{G})$ was assessed by quantitative realtime RT-PCR or western blotting. Total protein was extracted from BMDMs $6 \mathrm{~h}$ after administration of vehicle or LPS (100 $\mathrm{ng} / \mathrm{mL}$ ) plus a signaling inhibitor $(5 \mu \mathrm{M}$ BAY11-7082, $10 \mu \mathrm{M}$ SP600125, $10 \mu \mathrm{M}$ SB203580, $10 \mu \mathrm{M}$ U0126, $10 \mu \mathrm{M}$ LY2940002, or 20 mM NAC). Expression of mitoNEET protein was assessed by western blotting $(\mathbf{H})$. All data are expressed as the mean $\pm \mathrm{SD}$ from three independent experiments. ${ }^{*} P<$ 0.05 for vehicle vs. LPS treatment.

Initially, mitoNEET was identified as a mitochondrial target of thiazolidinediones such as pioglitazone and rosiglitazone, a peroxisome proliferator-activated receptor gamma (PPAR- $\gamma$ ) agonist, a class of medicines used to treat type-2 diabetes [28, 29]. Thiazolidinediones show antioxidative and anti-inflammatory activity in different disease models, including sepsis [30-32]. Overproduction of ROS during sepsis is thought to be a central part of the disease process $[6,33]$. However, the role of mitoNEET in sepsis is unknown. Here, we have used a mitoNEET ligand (NL1), modified TZD as a weaker affinity for PPARY $[15,28,34]$ and show that inhibiting expression or activity of mitoNEET reduces inflammation and oxidative stress during inflammatory responses and sepsis.

\section{RESULTS}

\section{Inflammatory stimuli induce expression of mitoNEET during} sepsis

Mitochondrial damage or dysfunction is the major cause of the multiple organ failure during sepsis [33]. Prior studies show that mitoNEET, an outer mitochondrial membrane protein, plays an important role in regulating mitochondrial function, especially oxidative capacity $[25,31,35]$. In this study, we hypothesized that mitoNEET plays a role in inflammation and oxidative stress during sepsis. To identify the role of mitoNEET during sepsis, we assayed expression of mitoNEET after induction of sepsis. Wild-type mice on a pure C57BL/6 genetic background were subjected to cecal ligation and puncture (CLP) to induce polymicrobial peritonitis, bacteremia, and sepsis. We then examined expression of mitoNEET mRNA and protein in the spleen $48 \mathrm{~h}$ later (Fig. 1A, B). Expression of mitoNEET mRNA and protein increased significantly (by 4.3-fold (Fig. 1A) and 4.1-fold (Fig. 1B), respectively) in mice with CLP-induced sepsis compared with sham mice $(n=4$ per group). In addition, expression of mitoNEET mRNA and protein increased by 2.2 -fold (Fig. 1C) and 3.4-fold (Fig. 1D), respectively, in mice with Escherichia coli (Gram-stain negative)-induced sepsis compared with sham mice ( $n=4$ per group). These data suggest that mitoNEET plays a critical role in sepsis caused by Gramnegative bacteria. To investigate the significance of mitoNEET induction by Gram-negative bacteria, we assessed its mRNA and protein levels in the spleen after induction of lipopolysaccharide (LPS)-mediated sepsis. LPS is a common pathogenic component of the outer membrane of Gram-negative bacteria. We harvested total RNA and protein from the spleen at $6,12,24,48$, and $72 \mathrm{~h}$ 

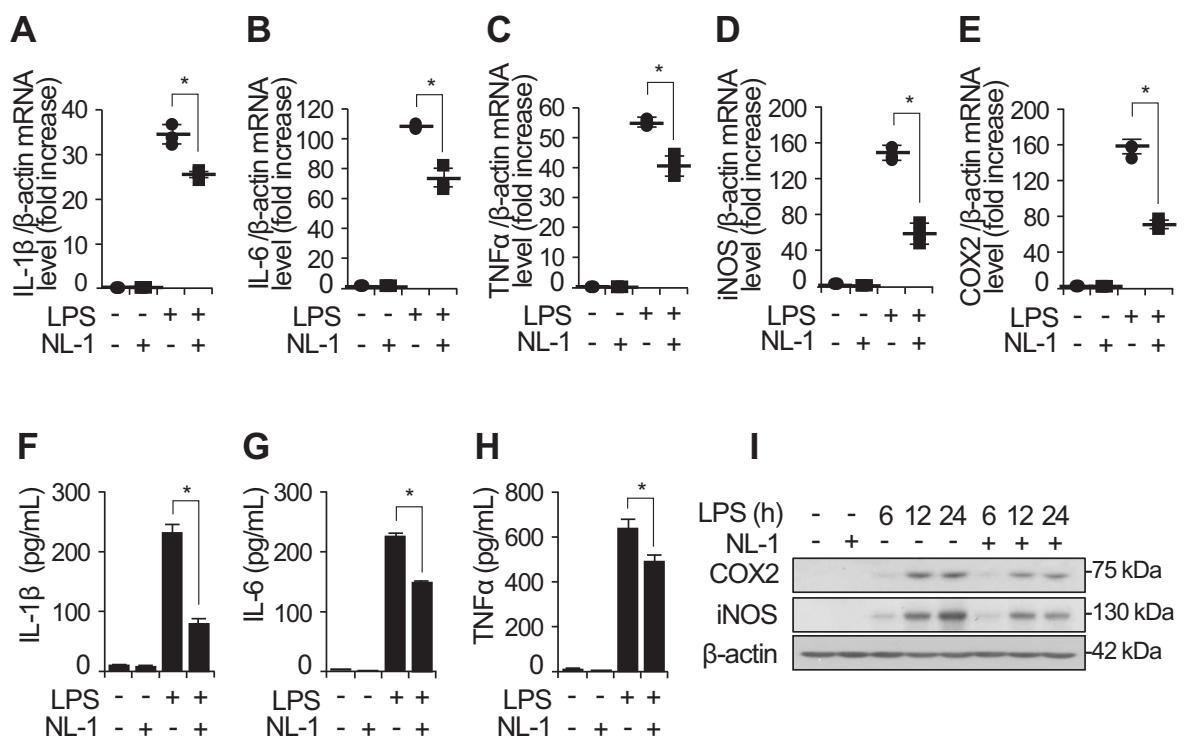

Fig. 2 Expression of inflammatory mediators decreases in the presence of the mitoNEET inhibitor NL-1. Total RNA, cell supernatants, and total protein were harvested from RAW264.7 cells $12 \mathrm{~h}$ (for mRNA) and $24 \mathrm{~h}$ (for protein) after treatment with vehicle, NL-1 (20 $\mu M$ ), LPS $(100 \mathrm{ng} / \mathrm{mL})$, or LPS plus NL-1 $(20 \mu \mathrm{M})$. Levels of mRNA encoding pro-inflammatory mediators IL-1 $\beta$, IL-6, TNF $\alpha$, iNOS, and COX2 were measured by quantitative real-time RT-PCR (A-E). Protein levels of pro-inflammatory mediators were analyzed using ELISA or western blotting $(\mathbf{F}-\mathbf{I})$. All data are presented as the mean \pm SD from three independent experiments. ${ }^{*} P<0.05$ for LPS vs. LPS plus NL-1.

after LPS injection. We found that mitoNEET mRNA levels began to increase by $6 \mathrm{~h}$ after LPS injection, and marked induction of mitoNEET was evident at $24 \mathrm{~h}$ (compared with vehicle) (Fig. 1E); protein levels increased at $48 \mathrm{~h}$ (Fig. 1F). Furthermore, expression of mitoNEET protein in bone marrow-derived macrophages (BMDMs) increased in the presence of LPS compared with vehicle (Fig. 1G). To identify the signaling pathway involved in regulating mitoNEET expression in LPS-stimulated macrophages, we used specific inhibitors Bay 11-7085 (an NF-kB inhibitor), SP600125 (a JNK MAP kinase inhibitor), SB203580 (a p38 MAP kinase inhibitor), U0126 (a mitogen-activated protein kinase kinase 1/2 (MEK1/2) inhibitor), LY294002 (a PI3 kinase inhibitor), and NAC (N-acetyl-Lcysteine, cytosolic ROS scavenger, A7250). BMDMs were treated with these kinase inhibitors in the presence of LPS, and levels of mitoNEET protein were assessed $6 \mathrm{~h}$ later. Bay 11-7085 blocked LPS-induced mitoNEET expression (Fig. 1H); however, the other inhibitors had no effect. These data suggest that mitoNEET may have a critical role in inflammation during sepsis, and that the LPSinduced NF-kB signaling pathway is involved in induction of mitoNEET expression under inflammatory conditions.

\section{Inhibition of mitoNEET reduces inflammatory responses during LPS stimulation of macrophages}

Macrophages play critical roles in various inflammatory diseases through release of inflammatory mediators and cytokines such as IL-1 $\beta, I L-6$, and TNFa. To investigate the role of mitoNEET during inflammatory responses, we analyzed LPS-stimulated expression of cytokines and mediators by RAW264.7 cells in the presence or absence of a mitoNEET inhibitor, mitoNEET Ligand-1 (NL-1), which was derived from glitazones [28] (Fig. 2). We found that NL-1 reduced expression of mRNA encoding pro-inflammatory cytokines IL-1 $\beta$, IL-6, and TNFa, and of mRNA encoding inflammatory mediators iNOS and COX2, in cells exposed to LPS for $12 \mathrm{~h}$ (Fig. $2 \mathrm{~A}-\mathrm{E}$ ). This decrease was not seen in control cells (treated with LPS alone). In addition, IL-1 $\beta$, IL-6, TNFa, iNOS, and COX2 protein levels decreased in the presence of NL-1 (Fig. 2F-I).

To investigate whether LPS-induced expression of mitoNEET alters inflammatory responses, we generated mitoNEET shRNA or control shRNA-expressing cells. Real-time PCR and western blot analyses were performed to assess expression of mitoNEET mRNA and protein, respectively (Fig. 3A, B). Expression of mRNA encoding IL-1 $\beta, I L-6$, TNFa, iNOS, and COX2 decreased in mitoNEET shRNA-expressing cells compared with control shRNA-expressing cells (Fig. 3C-G). This was also the case for protein expression (Fig. $3 \mathrm{H}-\mathrm{K})$. These data indicate that LPS-stimulated expression of mitoNEET is involved in inflammatory responses by macrophages via release of pro-inflammatory cytokines and mediators.

To assess the effect of mitoNEET inhibition during LPS-induced sepsis, we injected NL-1 intraperitoneally into wild-type C57BL/6 mice $12 \mathrm{~h}$ prior to injection of LPS. Blood was collected from the right atrium $48 \mathrm{~h}$ after LPS injection, and the concentration of IL-6 and TNFa in serum was measured. Control mice received vehicle alone. The levels of IL- 6 and TNFa fell markedly in the presence of NL-1 (Fig. 4A, B). Liver- and spleen-mediated immune responses are responsible for clearing bacteria and toxins, but they can also cause inflammation and organ damage [36, 37]. We found that NL1 reduced expression of mRNA encoding iNOS (Fig. 4C) and COX2 (Fig. 4D) in the spleen and liver of mice with LPS-induced sepsis. Taken together, these data demonstrate that mitoNEET is a key regulator of inflammatory responses during LPS-induced sepsis.

\section{Inhibition of mitoNEET attenuates LPS-induced oxidative stress and mitochondrial dysfunction}

Inflammatory processes induce oxidative stress and alter mitochondrial function [12]. When cells are under oxidative stress, mitoNEET acts as a redox-sensitive protein to induce transfer of the $[2 \mathrm{Fe}-2 \mathrm{~S}]$ cluster in mitochondria and plays a role in production of ROS [25]. Therefore, we analyzed whether inhibiting mitoNEET protects RAW264.7 cells from LPS-induced oxidative stress and mitochondrial dysfunction. Cells were treated with LPS in the presence or absence of NL-1 for $24 \mathrm{~h}$. Next, total ROS and superoxide were assayed by flow cytometry using a ROS/Superoxide Detection kit. LPS-induced total ROS and superoxide fell in the presence of $\mathrm{NL}-1$ (Fig. 5A, B). Furthermore, to investigate whether downregulation of mitoNEET regulates oxidative stress, we stimulated control shRNA- or mitoNEET shRNA-expressing RAW264.7 cells for $12 \mathrm{~h}$ with LPS $(1 \mu \mathrm{g} / \mathrm{mL})$, and measured total ROS and superoxide levels by flow cytometry. Total ROS and superoxide levels fell in LPS-treated cells expressing mitoNEET shRNA (Fig. 5C, D). In addition, confocal microscopy clearly 

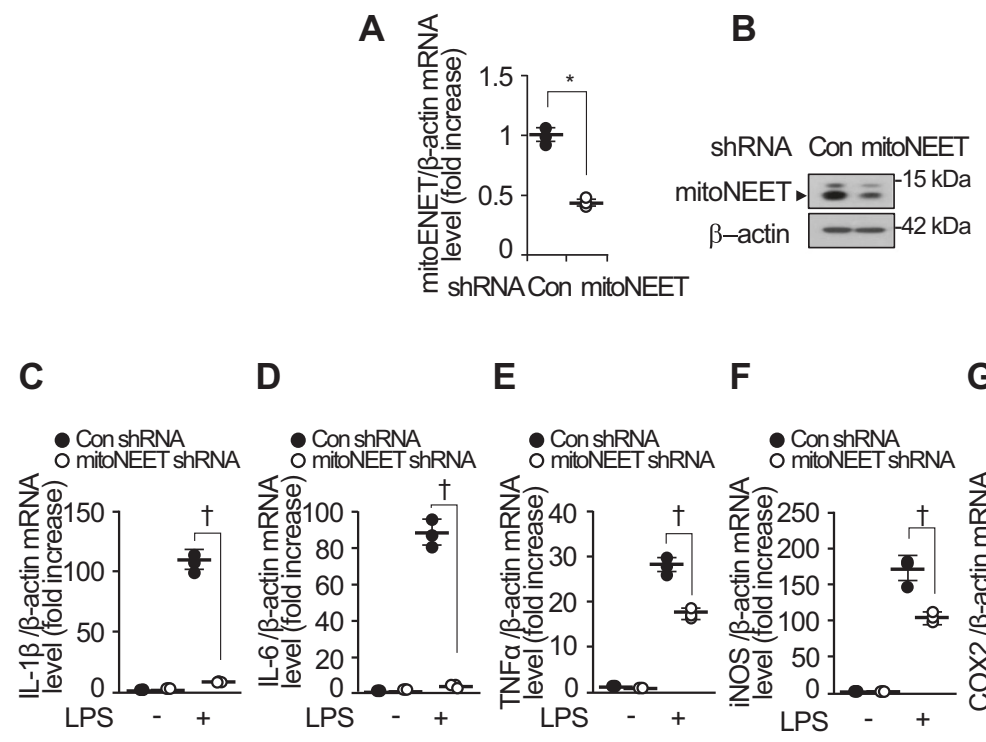

E $\quad F$

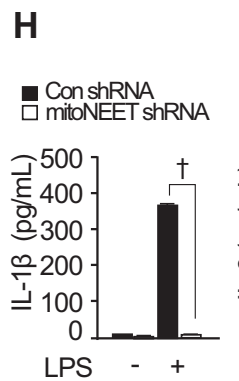

I

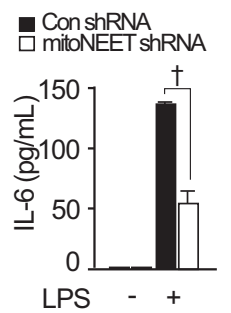

J
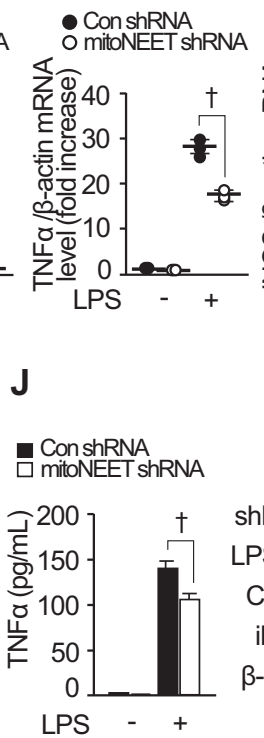

G

$\mathbf{F}$
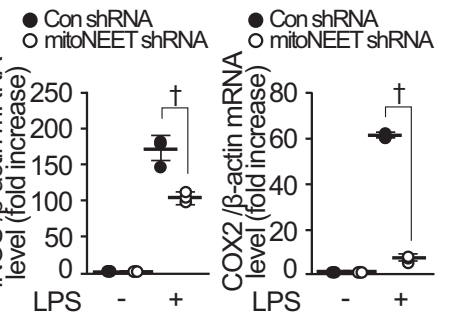

Fig. 3 Expression of mitoNEET shRNA decreases the levels of inflammatory mediators produced by RAW264.7 cells. RAW264.7 cells were transfected with control shRNA or mitoNEET shRNA and then subjected to RT-PCR or western blotting to verify downregulation of mitoNEET expression (A and B). $\beta$-actin was used as a loading control. RAW264.7 cells transfected with control shRNA or mitoNEET shRNA were stimulated with LPS $(100 \mathrm{ng} / \mathrm{mL})$ or vehicle. Total RNA, cell supernatants, and total protein were harvested $12 \mathrm{~h}$ (for mRNA) or $24 \mathrm{~h}$ (for protein) later. Expression of mRNA encoding IL-1 $\beta, \mathrm{IL}-6, \mathrm{TNF} \alpha$, iNOS, and COX2 was assessed by quantitative real-time RT-PCR (C-G), and protein expression was analyzed by ELISA or western blotting $(\mathbf{H}-\mathbf{K})$. All data are expressed as the mean \pm SD from three independent experiments. ${ }^{*} P<0.05$ for control shRNA-expressing cells vs. mitoNEET shRNA-expressing cells. ${ }^{\dagger} P<0.05$ for control shRNA-expressing cells vs. mitoNEET shRNA-expressing cells in the presence of LPS.

demonstrated that NL-1 or mitoNEET shRNA suppressed LPSinduced cytosolic ROS when compared with LPS alone (Fig. 5E, F). Treatment of cells with the iron chelator DFO (deferoxamine) in the presence of LPS showed results comparable to those observed after mitoNEET inhibition by NL-1 or mitoNEET shRNA (Fig. 5E, F). To verify whether inhibition of mitoNEET regulates mitochondrial dysfunction, we examined the mitochondrial membrane potential (MMP), a hallmark of mitochondrial dysfunction. Cells were treated $\mathrm{NL}-1$ in the presence or absence of LPS, and the loss of MMP was measured by flow cytometry using MitoProbe JC-1. Inhibition of mitoNEET rescued LPS-induced depolarization of the mitochondrial membrane (Fig. 6A). LPS-induced loss of MMP was also rescued by mitoNEET shRNA (Fig. 6B). To verify the effects of NL-1 on the MMP, we stained LPS-stimulated RAW264.7 cells with mitochondrial probes MitoTracker Red CMXRos, DiOC6(3), or TMRM (tetramethylrhodamine, methyl ester) in the presence or absence of NL-1. TMRM staining is used widely to monitor MMP. The MMP in LPS-treated cells fell but was rescued by NL-1 (Fig. 6C). DFO showed similar effects. To verify that inhibition of mitoNEET decreases the mitochondrial iron content, we stained mitochondrial iron using the mitochondrial probes Mitochondrial Marker Deep Red and Mito-ferroGreen by, which allow visualization of ferrous ion $\left(\mathrm{Fe}^{2+}\right)$ by confocal microscopy. We found that $\mathrm{NL}-1$ depleted mitochondrial $\mathrm{Fe}^{2+}$ in the presence of LPS. Similar results were obtained using DFO (Fig. 6D). Taken together, these data suggest that inhibiting mitoNEET in RAW264.7 cells reduces mitochondrial iron content, thereby preventing oxidative stress and mitochondrial dysfunction during LPS-induced inflammation.

Oxidant-induced injury during inflammatory processes such as sepsis induces organ failure [10]. To confirm the anti-inflammatory effects of NL-1 during LPS-induced oxidative stress, we examined expression of $\mathrm{HO}-1$ (heme oxygenase-1), SOD2 (superoxide dismutase 2), and SOD1 (superoxide dismutase 1) mRNA and protein in LPS-stimulated RAW264.7 cells in the presence or absence of NL-1 (Fig. 7A-D). NL-1 increased expression of HO-1 and SOD2 mRNA and protein, but not that of SOD1 mRNA and protein. Consistent with this, HO-1 and SOD2 mRNA and protein levels in cells expressing mitoNEET shRNA were higher than those in control shRNA-expressing cells (Fig. 7E-H). These results demonstrate that NL-1 or mitoNEET shRNA may attenuate oxidative-induced organ injury during LPS-induced inflammation by upregulating expression of antioxidant-defense genes.

\section{DISCUSSION}

Sepsis is one of the most serious causes of mortality worldwide. There is increasing evidence that oxidative stress plays a major role in organ dysfunction by driving excessive inflammation [13, 38, 39]. Inflammation-induced ROS production promotes dysfunction of mitochondria, a major site of ROS production, thereby activating 
A

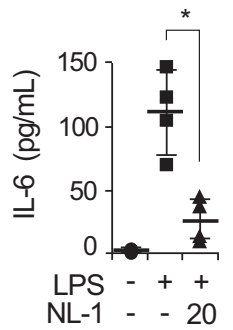

B

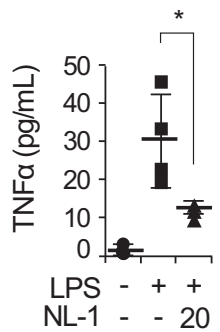

C

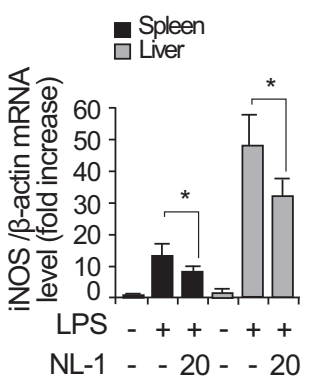

D

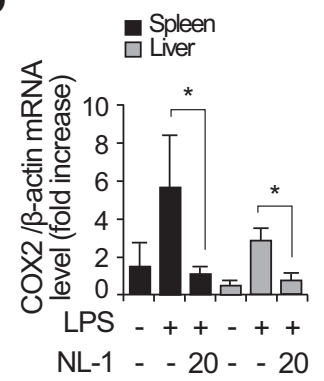

Fig. 4 Expression of inflammatory mediators triggered by LPSinduced sepsis was diminished in the presence of a mitoNEET inhibitor. C57BL/6 mice were injected intraperitoneally with vehicle, LPS $(20 \mathrm{mg} / \mathrm{kg})$, or LPS plus NL-1 $(20 \mathrm{mg} / \mathrm{kg})$. Blood was collected from the right atrium $48 \mathrm{~h}$ later, and IL- 6 and TNF $\alpha$ levels in serum were measured (A and $\mathbf{B}$ ). Total RNA was harvested from the spleen and liver. Expression of mRNA encoding iNOS and COX2 was assessed in the spleen or liver (C and $\mathbf{D}$ ) by quantitative real-time RTPCR. For all real-time PCR analyses, $\beta$-actin was used as a control for normalization. All data are expressed as the mean \pm SD from three independent experiments. ${ }^{*} P<0.05$ for LPS vs. LPS plus NL-1 treatment.

oxidative stress and generating a self-feeding cycle [11, 40]. Thus, a therapeutic strategy targeting mitochondrial dysfunction has the potential to break this vicious cycle and prevent progression of oxidative stress and sepsis [13]. Recent studies suggest that iron is an essential component of cellular processes such as mitochondrial energy metabolism; however, mitochondrial iron overload is a major cause of mitochondrial damage and $\operatorname{ROS}[13,41,42]$. In this study, we demonstrated that inhibiting mitoNEET, a mitochondrial iron regulator, has a protective effect against inflammatory responses during sepsis. MitoNEET, a mitochondrial protein, plays a key role in energy metabolism, iron regulation, and production of ROS by mitochondria $[19,43]$. Our data show that inflammatory stimuli, such as LPS, which induce mitochondrial oxidative damage, trigger production of mitoNEET mRNA and protein in various animal models of and in BMDMs (Fig. 1). Furthermore, the LPS-induced NF$\mathrm{kB}$ signaling pathway is involved in induction of mitoNEET expression during inflammatory conditions (Fig. $1 \mathrm{H}$ ). These data suggest that mitoNEET could play a key role in energy metabolism as well as in inflammation. Interestingly, expression of inflammatory mediators IL-1 $\beta, I L-6$, TNFa, iNOS, and COX2 decreased in the presence of a mitoNEET inhibitor, NL-1, or upon expression of mitoNEET shRNA, even in the LPS-induced sepsis model (Figs. 2, 3, and 4). MitoNEET was first identified as a redox-sensitive mitochondrial target of the thiazolidinedione (TZD) pioglitazone $[28,29]$. Earlier studies observed that overexpression of mitoNEET inhibits mitochondrial iron transport to the matric and reduces ROSmediated damage [21]. This led to a reduction in the MMP and accumulation of ROS in mouse adipocytes [22]. Conversely, we found that ROS levels, MMP, and iron content were reduced in the presence of NL-1 or mitoNEET shRNA under inflammatory conditions (Figs. 5 and 6). Furthermore, expression of the antioxidant enzyme HO-1 and the mitochondrial MnSOD isoform SOD2 was

enhanced in the presence of NL-1 or mitoNEET shRNA after LPS administration; however, expression of SOD1, a major cytoplasmic antioxidant enzyme, was not enhanced. The reason for these different results could be because mitoNEET acts differently according to the redox conditions in a cell. The biophysical properties of mitoNEET involve electron and Fe-S cluster transfer $[17,44,45]$. In a reducing environment, mitoNEET is incapable of [2Fe-2S] cluster transfer; thus accumulation of iron in the mitochondria is abrogated by accelerating loss of the [2Fe-2S] cluster [46]. However, only when cells are under oxidative stress does mitoNEET [2Fe-2S] transfer [2Fe-2S] clusters to apoproteins, and electrons from NADH to oxygen or ubiquinone, in mitochondria $[18,19,25]$. In an oxidizing environment, mitoNEET contributes to oxidative stress and production of superoxide radicals $\left(\mathrm{O}_{2}{ }^{-}\right)$by transferring iron to the mitochondrial matrix and electrons to oxygen through oxidation of NADH (the electron donor). Recent publications demonstrated that pioglitazone stabilizes the $2 \mathrm{Fe}-2 \mathrm{~S}$ cluster and inhibits iron transfer from mitoNEET to mitochondria. Pioglitazone, which shows strong preferential binding to mitoNEET in the oxidized state, may therefore act to alleviate stress caused by Fe overload [25]. These data suggest that iron regulation via targeting of mitoNEET rescues ROS production and mitochondrial dysfunction in the oxidized state $[41,47,48]$. Therefore, our results demonstrate that mitoNEET is a possible therapeutic molecule for mitochondrial dysfunction during inflammatory diseases and sepsis.

\section{MATERIALS AND METHODS}

\section{Cell culture and reagents}

RAW264.7 cells were cultured in Dulbecco Modified Eagle Medium (Life Technologies, Grand Island, NY, USA), 5\% fetal bovine serum, 100 units $/ \mathrm{mL}$ penicillin, and $100 \mathrm{mg} / \mathrm{mL}$ streptomycin under an atmosphere of $95 \%$ air and $5 \% \mathrm{CO}_{2}$ at $37^{\circ} \mathrm{C}$. Bone marrow-derived macrophages (BMDMs) from C57BL/6 mice were isolated and differentiated as described previously [49]. Briefly, bone marrow cells $\left(3 \times 10^{7}\right.$ cells) were cultured in macrophage-differentiation medium with GM-CSF at $37{ }^{\circ} \mathrm{C}$ for 7 days. The adherent macrophages were detached from culture dishes by treatment with 5\% EDTA in PBS, followed by scraping with a sterile cell scraper. The resuspended cells were then directly seeded on cell culture plates for other experiments.

mitoNEET inhibitor, NL-1, was purchased from (Merck Millipore, Billerica, MA, USA, 475825). Deferoxamine mesylate salt (DFO) was purchased from (Sigma-Aldrich, St Louis, MO, D9533). Lipopolysaccharides from Escherichia coli O26:B6, $\gamma$-irradiated, BioXtra, suitable for cell culture (Sigma-Aldrich, St Louis, MO, L2654).

\section{Animal experiments}

Animal care and use for all experiments were approved from the animal facilities at University of Ulsan (SWC-14-012). C57BL/6 mice were purchased from ORIENT BIO Inc (Busan, Korea). CLP-induced polymicrobial sepsis and fibrin clot experiment were performed as previously described [50]. Using sterile conditions, the fibrin clot containing E. coli or S. aureus $\left(1 \times 10^{8} \mathrm{CFU}\right)$ was placed within the peritoneal cavity of C57BL/6 mice. Lipopolysaccharides from $E$. coli 0127 :B8 - purified by phenol extraction were purchased from (Sigma-Aldrich, St Louis, MO, L3129) for LPS-induced sepsis. mitoNEET inhibitor, NL-1 $(20 \mathrm{mg} / \mathrm{kg})$, was administrated to C57BL/6 mice $12 \mathrm{~h}$ before LPS $(20 \mathrm{mg} / \mathrm{kg})$ injection (pre-administration).

\section{Western immunoblotting and enzyme-linked immunosorbent assay \\ Total protein isolation and Western blotting were performed as described previously [ref]. The blots were incubated with antibody an anti-CISD1 (1:2000) (Protein Tech, 16006-1-AP), an anti- $\beta$-actin (1:5000) (Sigma-Aldrich, St Louis, MO, A5441), an anti-phospho-lkBa (1:1000) (Santa Cruz Biotechnology, sc-8404), an anti-lkBa (1:1000) (Santa Cruz Biotechnology, sc-847) and an anti-COX2 (1:1000) (Cayman chemical, 160106), an anti- iNOS (1:1000) (Santa Cruz Biotechnology, sc-650) and an anti-HO-1 (1:3000) (Enzo Life Sciences, ADI-SPA-896), an anti-SOD2 (1:3000) (Santa Cruz Biotechnology, sc-30080), an anti-SOD1 (1:1000) (Santa Cruz Biotechnol- ogy, sc-11407) and anti-NLRP3/NALP3 (1:1000) (Adipogen, AG-20B-0014- C100), an-anti-P2X7R (1:3000) (Alomone Labs, APR-004) in TBST overnight at room temperature. The blots were incubated with an anti-secondary}


A

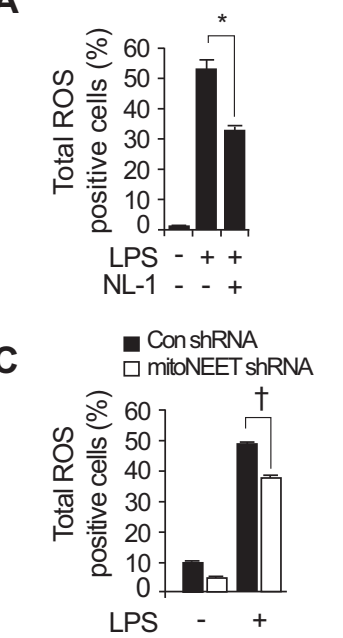

B

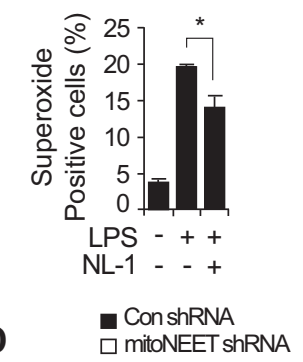

mitoNEET shRNA
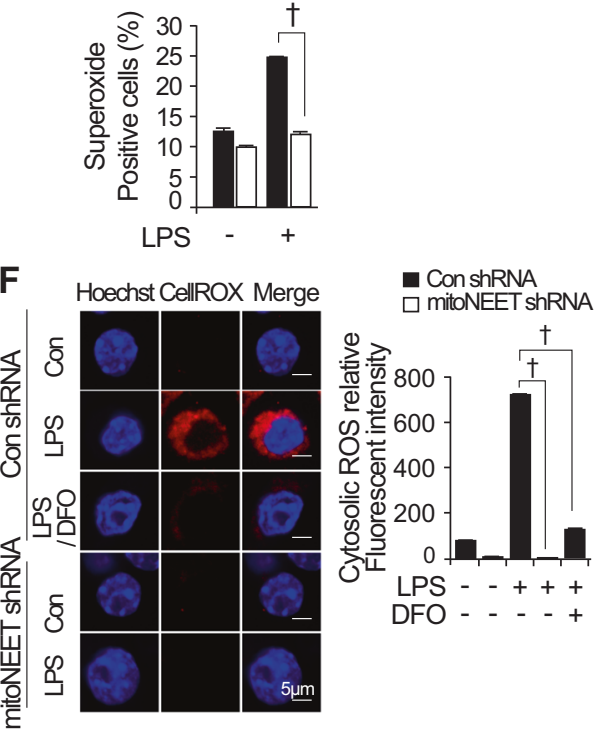

Fig. 5 LPS-induced reactive oxygen species and mitochondrial dysfunction are attenuated by inhibition of mitoNEET. RAW264.7 cells expressing control shRNA or mitoNEET shRNA were treated with vehicle, LPS ( $1 \mu \mathrm{g} / \mathrm{mL})$, or LPS plus NL-1 (20 $\mu \mathrm{M})$ for $24 \mathrm{~h}$. Total reactive oxygen species (ROS) and superoxide anions were assayed by flow cytometry using a ROS/Superoxide Detection kit (A-D). All data shown are expressed as the mean \pm SD from three independent experiments. ${ }^{*} P<0.05$ for LPS vs. LPS plus NL- 1 treatment; ${ }^{\dagger} P<0.05$ for control shRNAexpressing cells vs. mitoNEET shRNA-expressing cells in the presence of LPS. ${ }^{*} P<0.05$ indicates a significant decrease compared with vehicle; ${ }^{\dagger} P<0.05$ indicates significant decrease compared with control siRNA. RAW264.7 cells expressing control shRNA or mitoNEET shRNA were treated with vehicle or LPS $(1 \mu \mathrm{g} / \mathrm{mL})$ for $12 \mathrm{~h}$. Cells were then stained with the fluorescent probes Hoechst 33258 (nuclei, blue) and CellROX ${ }^{\circ}$ Deep Red (total ROS, red). Immunofluorescence images of cells stained with Hoechst 33258 (nuclei, blue) and CellROX Deep Red (total ROS, red) (E and F). Scale bar: $5 \mu \mathrm{m}$. Fluorescence intensity was measured using image analysis software (ImageJ). All data are expressed as the mean \pm SD from three independent experiments. ${ }^{*} P<0.05$ for LPS vs. LPS plus NL-1 or DFO. ${ }^{\dagger} P<0.05$ for control shRNA-expressing cells vs. mitoNEET shRNA-expressing cells in the presence of LPS or vs. LPS plus DFO.

antibody (1:5000) in TBST, immunoblots were detected by SuperSignal ${ }^{\circ}$ West Pico Chemiluminescent Substrate (Pierce) and visualized after exposure to X-ray film.

Mouse IL-1 $\beta$ (R\&D systems, Minneapolis, MN, USA, DY401), IL-6 (R\&D systems, Minneapolis, MN, USA, DY406), and TNFa (R\&D systems, Minneapolis, MN, USA, DY410) were measured from cell culture supernatant of BMDMs and RAW264.7 cells using enzyme-linked immunosorbent assay (ELISA).

\section{Quantitative real-time reverse transcription-polymerase chain reaction (qRT-PCR)}

Total RNA was isolated TRIzol reagent (Invitrogen, Life technologies, Carlsbad, CA), Reverse transcription was performed using SuperScript ${ }^{\mathrm{TM}}$ III First-Strand Synthesis System (Invitrogen, Carlsbad, CA). Real-time quantitative PCR was conducted using iQ SYBR Green Supermix (Bio-Rad, Hercules, CA). Triplicate samples per condition were analyzed on an Applied Biosystems StepOnePlusTM Real-Time PCR System using absolute quantification settings. The primers sequences were as follows: mouse mitoNEET (forward: 5'-CAA GGC TAT GGT GAA TCT TCA G-3' and reverse: $5^{\prime}$-GTG CCA TTC TAC GTA AAT CAG-3'), mouse $\beta$-actin (forward: $5^{\prime}$-GAT CTG GCA CCA CAC CTT CT-3' and reverse: $5^{\prime}-\mathrm{GGG}$ GTG TTG AAG GTC TCA AA-3'). Mouse IL-1 $\beta$ (forward: $5^{\prime}-$ TTG ACG GAC CCC AAA AGA TG-3' and reverse: $5^{\prime}-$ AGA AGG TGC TCA TGT CCT CA-3'), mouse IL-6 (forward: $5^{\prime}$-GAG GAT ACC ACT CCC AAC AGA CC- $3^{\prime}$ and reverse: $5^{\prime}$-AAG TGC ATC ATC GTT GTT CAT ACA-3'), mouse TNFa (forward: 5'- GCC TCT TCT CAT TCC TGC TTG-3' and reverse: 5'-CTG ATG AGA GGG AGG CCA TT-3'), mouse IL-6 (forward: 5'-
GAG GAT ACC ACT CCC AAC AGA CC- $3^{\prime}$ and reverse: $5^{\prime}$ - AAG TGC ATC ATC GTT GTT CAT ACA-3'), and mouse COX2 (forward: 5'-CAA GGG AGT CTG GAA CAT TG-3' and reverse: $5^{\prime}$-ACC CAG GTC CTC GCT TAT GA-3'), mouse iNOS (forward: $5^{\prime}-A A C$ GGA GAA CGT TGG ATT TG-3' and reverse: $5^{\prime}-\mathrm{CAG}$ CAC AAG GGG TाT TCT TC-3'), mouse HO-1 (forward: $5^{\prime}$-CGC CTT CCT GCT CAA CAT T-3' and reverse: $5^{\prime}$-TGT GTT CCT CTG TCA GCA TCA C-3'), mouse SOD2 (forward: 5'- ATG GTG GGG GAC ATA TT-3' and reverse: 5'-GAA CCT TGG ACT CCC ACA GA $\left.-3^{\prime}\right)$. Amplification of CDNA started with $10 \mathrm{~min}$ at $95^{\circ} \mathrm{C}$, followed by 40 cycles of $15 \mathrm{~s}$ at $95^{\circ} \mathrm{C}$ and $1 \mathrm{~min}$ at $60^{\circ} \mathrm{C}$.

\section{Construction of mitoNEET shRNA-expressing cells}

The mitoNEET shRNA and the nonspecific control shRNA (Sigma-Aldrich, St Louis, MO) were transfected into RAW264.7 cells using transfection reagents (Promega, Madison, WI, USA) according to the manufacturer's protocol. The sequences of mouse mitoNEET shRNA were as follows: 5'-CCG GCG TAG GAC CTC TGA TCA TCA ACT CGA GTT GAT GAT CAG AGG TCC TAC GTT TाT TG-3'. The expression of mitoNEET and $\beta$-actin in stable cells was measured.

\section{Flow cytometry for total reactive oxygen species (ROS) and superoxide measurement}

Cells were treated with vehicle, LPS, LPS plus NL-1, or LPS plus DFO (desferoxamine). Total ROS and superoxide were detected using ROS-ID ${ }^{\circ}$ total ROS/Superoxide Detection kit (Enzo Life Sciences, Farmingdale, NY, USA, ENZ-51010) according to the protocol of the manufacturer. 
A

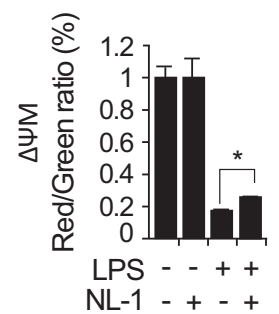

B

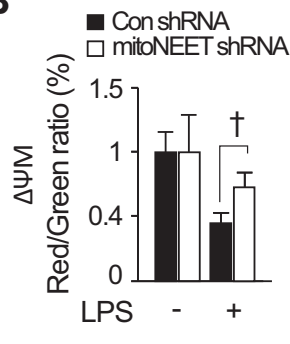

C
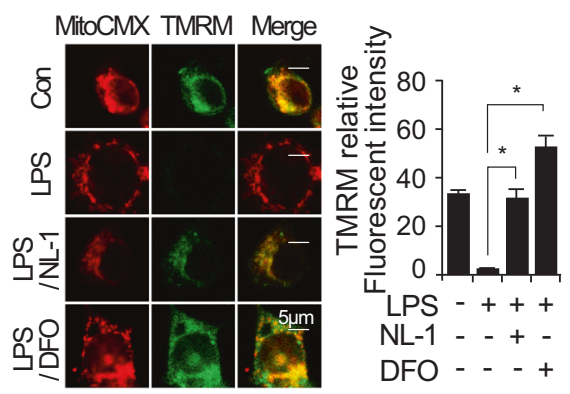
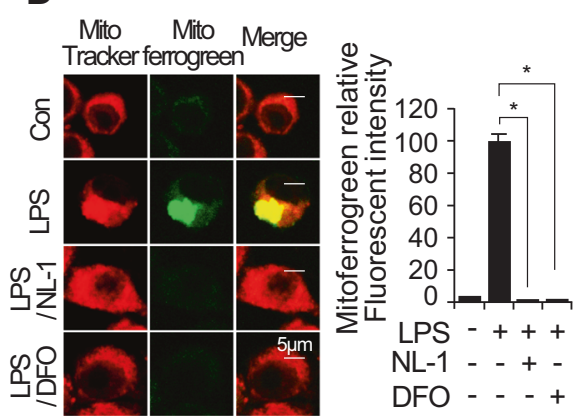

Fig. 6 LPS-induced ROS and mitochondrial dysfunction are activated by mitoNEET-mediated iron accumulation. RAW264.7 cells expressing control shRNA or mitoNEET shRNA were treated with vehicle, LPS $(1 \mu \mathrm{g} / \mathrm{mL})$, or LPS plus NL-1 (20 $\mu \mathrm{M})$ for $24 \mathrm{~h}$. The mitochondrial membrane potential (MMP) was measured by flow cytometry using MitoProbe JC-1 (A and B). The histogram shows the ratio of JC-1 polymer (red) to JC-1 monomer (green) fluorescence, which is an index of the MMP. A decrease in the red/green ratio indicates depolarization of the mitochondrial membrane. RAW264.7 cells expressing control shRNA or mitoNEET shRNA were treated with vehicle, LPS (1 $\mu \mathrm{g} / \mathrm{mL}), \mathrm{LPS}$ plus NL$1(20 \mu \mathrm{M})$, or LPS plus DFO $(500 \mu \mathrm{M})$ for $12 \mathrm{~h}$. Cells were then stained with MitoTracker Red CMXRos, DiOC6(3), (red), and TMRM (tetramethylrhodamine, methyl ester, Perchlorate, green). (C). Cells were stained with MitoTracker Deep Red (a mitochondrial marker; red) and Mito-FerroGreen (a marker of mitochondrial Fe2+; green) after treatment (D). Immunofluorescence images of MitoTracker Red CMXRos, DiOC6 (3), (mitochondria, red), TMRM (green), and MitoTracker Deep Red, Mito-FerroGreen (green) (C and D). Scale bar: $5 \mu \mathrm{m}$. Fluorescence intensity was measured using ImageJ. All data are expressed as the mean \pm SD from three independent experiments. ${ }^{*} P<0.05$ for LPS vs. LPS plus NL-1 or DFO. ${ }^{\dagger} P<0.05$ for control shRNA-expressing cells vs. mitoNEET shRNA-expressing cells in the presence of LPS.

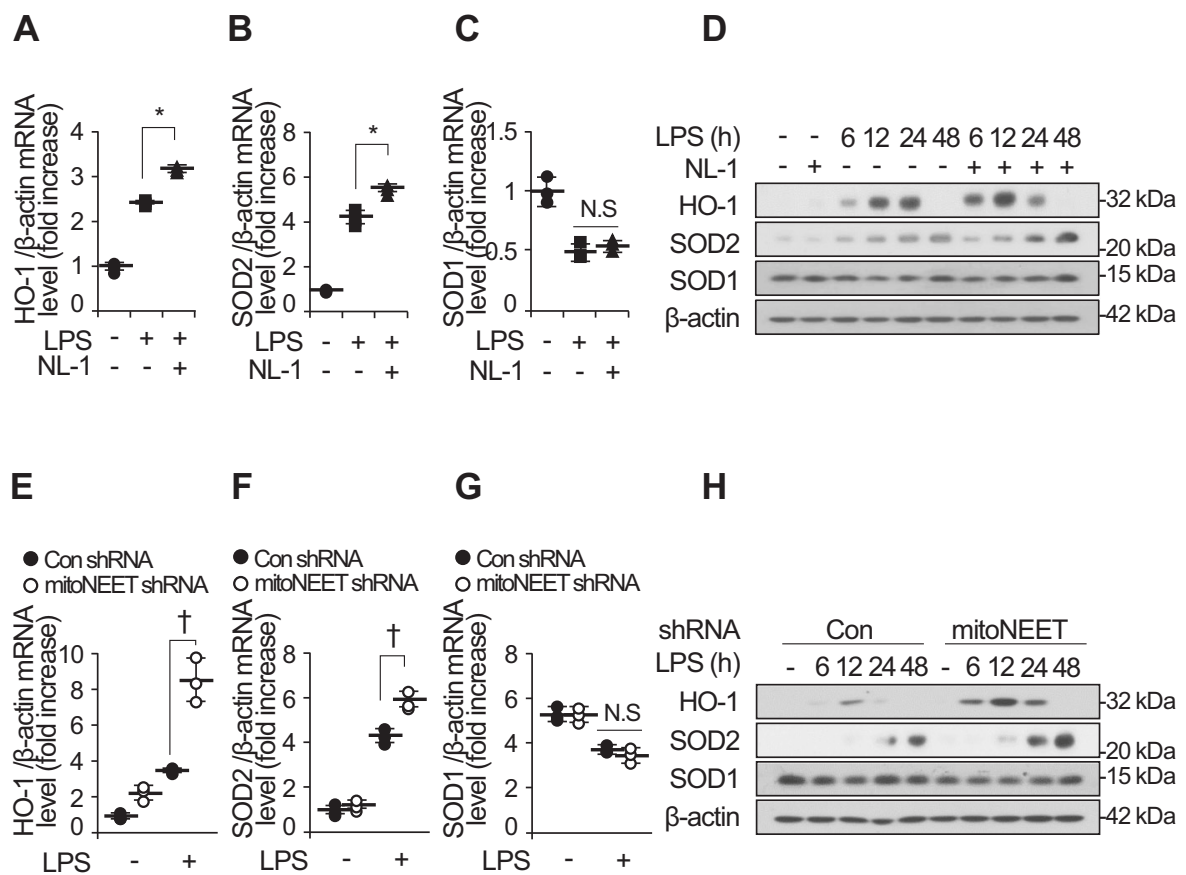

Fig. 7 Inhibition of mitoNEET increases expression of antioxidant defense genes. Control shRNA-expressing or mitoNEET-expressing RAW264.7 cells were treated with vehicle, LPS $(1 \mu \mathrm{g} / \mathrm{mL})$, or LPS plus NL-1 $(20 \mu \mathrm{M})$. Total RNA and protein were harvested $12 \mathrm{~h}$ later, followed by measurement of mRNA encoding HO-1, SOD2, or SOD1 by quantitative real-time RT-PCR (A-C and E-G). Expression of HO-1, SOD2, and SOD1 protein was detected by western blotting $(\mathbf{D}$ and $\mathbf{H})$. All data are expressed as the mean \pm SD from three independent experiments. ${ }^{*} P<0.05$ for LPS vs. LPS plus NL-1 treatment. ${ }^{\dagger} P<0.05$ for control shRNA-expressing cells vs. mitoNEET shRNA-expressing cells in the presence of LPS. NS, not significant. 
Measurements were performed on a FACS Calibur (Becton Dickinson, San Jose, CA, USA) flow cytometer.

\section{Flow cytometry for mitochondrial membrane potential measurements}

To measure the mitochondrial membrane potential, cells were stained using the MitoProbe JC-1 staining kits (MitoProbe ${ }^{\mathrm{TM}} \mathrm{JC}-1$ Assay Kit for Flow Cytometry, Thermo Fisher Scientific, Carlsbad, CA, USA, M34152) according to the protocol of the manufacturer. Measurements were performed on a FACSCalibur (Becton Dickinson, San Jose, CA, USA) flow cytometer (Ex = $488 \mathrm{~nm}$ and $\mathrm{Em}=590 \mathrm{~nm}$ for JC-1 aggregates; $\mathrm{Ex}=488 \mathrm{~nm}$ and $\mathrm{Em}=$ $529 \mathrm{~nm}$ for JC-1 monomers). Histogram of the percent of JC-1 red/green ratio calculated the relative ratio of red (JC-1 polymer) against green (JC-1 monomer) fluorescence.

\section{Confocal microscopy}

RAW264.7 cells were seeded at $1 \times 10^{5}$ cells per well on coverslips in 24well plates and treated with reagents. After reagent treatment, media was removed by washing with PBS and cells were incubated with serum free media containing $5 \mu \mathrm{M}$ Cell ROX red (CellROX ${ }^{\circ}$ Deep Red Reagent for oxidative stress detection, Invitrogen, Life Technologies, Carlsbad, CA, C10422) or 200 nM Mitotracker red CMXRos (Invitrogen, Life Technologies, Carlsbad, CA, M7512) and $500 \mathrm{nM}$ TMRM (tetramethylrhodamine, methyl ester, Perchlorate, Invitrogen, Life Technologies, Carlsbad, CA, T668) or $100 \mathrm{nM}$ Mitotracker red (Mitochondrial marker deep red, Invitrogen, Life Technologies, Carlsbad, CA, M22426) and $5 \mu \mathrm{M}$ Mito-FerroGreen (Dojindo Laboratories, Kumamoto, Japan, M489) for $30 \mathrm{~min}$ at $37^{\circ} \mathrm{C}$ in the dark. Then, cells fixed for $20 \mathrm{~min}$ in $4 \%$ formaldehyde, rinsed 3 times in PBS. A nuclear counterstaining was made with a solution of $1 \mu \mathrm{g} / \mathrm{mL}$ Hoechst 33258 stain for $5 \mathrm{~min}$ and mounting on a slide Fluorescence Mounting Medium (DAKO North America Inc, Carpinteria, CA, United States, S3023). Olympus FV1000 MPE microscope was used to acquire images.

\section{Statistical analysis}

All results were confirmed in at least three independent experiments; data from one representative experiment are shown. Quantitative data are shown as means \pm standard deviation and significance of statistical analysis was determined with two-tailed, unpaired Student's $t$-test. $P$ values $<0.05$ were considered significant.

\section{DATA AVAILABILITY}

All data needed to evaluate the conclusions in the paper are present in the paper. Additional data related to this paper may be requested from the corresponding author.

\section{REFERENCES}

1. Hotamisligil GS. Inflammation and metabolic disorders. Nature 2006;444:860-7.

2. Nedeva C, Menassa J, Puthalakath H. Sepsis: inflammation is a necessary evil. Front Cell Dev Biol. 2019;7:108.

3. Reale $\mathrm{M}$, Conti L, Velluto $\mathrm{D}$. Immune and inflammatory-mediated disorders: from bench to bedside. J Immunol Res. 2018;2018:7197931.

4. Chen X-H, Yin Y-J, Zhang J-X. Sepsis and immune response. World J Emerg Med. 2011;2:88-92.

5. Shenoy S. Coronavirus (Covid-19) sepsis: revisiting mitochondrial dysfunction in pathogenesis, aging, inflammation, and mortality. Inflamm Res. 2020;69:1077-85.

6. Víctor VM, Espulgues JV, Hernández-Mijares A, Rocha M. Oxidative stress and mitochondrial dysfunction in sepsis: a potential therapy with mitochondriatargeted antioxidants. Infect Disord Drug Targets 2009;9:376-89.

7. Murphy MP. How mitochondria produce reactive oxygen species. Biochem J. 2009;417:1-13.

8. Levi S, Rovida E. The role of iron in mitochondrial function. Biochim Biophys Acta. 2009;1790:629-36.

9. Li Y, Hongwei Y. Mitochondrial dysfunction in inflammatory responses and cellular senescence: pathogenesis and pharmacological targets for chronic lung diseases. Br J Pharmacol. 2016;173:2305-18.

10. Nagar H, Piao S, Kim C-S. Role of mitochondrial oxidative stress in sepsis. Acute Crit Care. 2018;33:65-72.

11. Urrutia PJ, Mena NP, Núñez MT. The interplay between iron accumulation, mitochondrial dysfunction, and inflammation during the execution step of neurodegenerative disorders. Front Pharm. 2014;5:38.
12. López-Armada MJ, Riveiro-Naveira RR, Vaamonde-García C, Valcárcel-Ares MN. Mitochondrial dysfunction and the inflammatory response. Mitochondrion. 2013;13:106-18.

13. Zhang $\mathrm{H}$, Feng $\mathrm{Y}-\mathrm{W}$, Yao $\mathrm{Y}-\mathrm{M}$. Potential therapy strategy: targeting mitochondrial dysfunction in sepsis. Mil Med Res. 2018;5:41.

14. Huang ML-H, Chiang S, Kalinowski DS, Bae D-H, Sahni S, Richardson DR. The role of the antioxidant response in mitochondrial dysfunction in degenerative diseases: cross-talk between antioxidant defense, autophagy, and apoptosis. Oxid Med Cell Longev. 2019;2019:6392763.

15. Geldenhuys WJ, Leeper TC, Carroll RT. mitoNEET as a novel drug target for mitochondrial dysfunction. Drug Disco Today. 2014;19:1601-6.

16. Paddock ML, Wiley SE, Axelrod HL, Cohen AE, Roy M, Abresch EC, et al. MitoNEET is a uniquely folded $2 \mathrm{Fe} 2 \mathrm{~S}$ outer mitochondrial membrane protein stabilized by pioglitazone. Proc Natl Acad Sci USA. 2007;104:14342-7.

17. Wiley SE, Paddock ML, Abresch EC, Gross L, van der Geer P, Nechushtai R, et al. The outer mitochondrial membrane protein mitoNEET contains a novel redoxactive 2Fe-2S cluster. J Biol Chem. 2007;282:23745-9.

18. Wang $\mathrm{Y}$, Landry AP, Ding $\mathrm{H}$. The mitochondrial outer membrane protein mitoNEET is a redox enzyme catalyzing electron transfer from FMNH 2 to oxygen or ubiquinone. J Biol Chem. 2017;292:10061-7.

19. Li X, Wang Y, Tan G, Lyu J, Ding H. Electron transfer kinetics of the mitochondrial outer membrane protein mitoNEET. Free Radic Biol Med. 2018;121:98-104.

20. Tasnim H, Landry AP, Fontenot $\mathrm{CR}$, Ding $\mathrm{H}$. Exploring the FMN binding site in the mitochondrial outer membrane protein mitoNEET. Free Radic Biol Med. 2020;156:11-19.

21. Kusminski CM, Holland WL, Sun K, Park J, Spurgin SB, Lin Y, et al. MitoNEETdriven alterations in adipocyte mitochondrial activity reveal a crucial adaptive process that preserves insulin sensitivity in obesity. Nat Med. 2012;18:1539-49.

22. Sohn Y-S, Tamir S, Song L, Michaeli D, Matouk I, Conlan AR, et al. NAF-1 and mitoNEET are central to human breast cancer proliferation by maintaining mitochondrial homeostasis and promoting tumor growth. Proc Natl Acad Sci USA 2013;110:14676-81.

23. Kusminski CM, Holland WL, Sun K, Park J, Spurgin SB, Lin Y, et al. MitoNEET, a key regulator of mitochondrial function and lipid homeostasis. Nat Med. 2012;18:1539-49.

24. Kusminski CM, Park J, Scherer PE. MitoNEET-mediated effects on browning of white adipose tissue. Nat Commun. 2014;5:3962.

25. John A, Harir Y, Conlan AR, Shvartsman M, Michaeli D, Tamir S, et al. Facile transfer of [2Fe-2S] clusters from the diabetes drug target mitoNEET to an apoacceptor protein. Proc Natl Acad Sci USA. 2011;108:13047-52.

26. Taminelli GL, Sotomayor V, Valdivieso AG, Teiber ML, Marín MC, Santa-Coloma TA. CISD1 codifies a mitochondrial protein upregulated by the CFTR channel. Biochem Biophys Res Commun. 2008;365:856-62.

27. Chen X-Y, Ren H-H, Wang D, Chen Y, Qu C-J, Pan Z-H, et al. Isoliquiritigenin induces mitochondrial dysfunction and apoptosis by inhibiting mitoNEET in a reactive oxygen species-dependent manner in A375 human melanoma cells. Oxid Med Cell Longev. 2019;2019:9817576.

28. Geldenhuys WJ, Funk MO, Barnes KF, Carroll RT. Structure-based design of a thiazolidinedione which targets the mitochondrial protein mitoNEET. Bioorg Med Chem Lett. 2010;20:819-23.

29. Colca JR, McDonald WG, Waldon DJ, Leone JW, Lull JM, Bannow CA, et al. Identification of small molecules that bind to the mitochondrial protein mitoNEET. Am J Physiol Endocrinol Metab. 2004;286:E252-60.

30. Zhu M, Flynt L, Ghosh S, Mellema M, Banerjee A, Williams E, et al. Antiinflammatory effects of thiazolidinediones in human airway smooth muscle cells. Am J Respir Cell Mol Biol. 2011;45:111-9.

31. Logan SJ, Yin L, Geldenhuys WJ, Enrick MK, Stevanov KM, Carroll RT, et al. Novel thiazolidinedione mitoNEET ligand-1 acutely improves cardiac stem cell survival under oxidative stress. Basic Res Cardiol. 2015;110:19.

32. Faine LA, Rudnicki M, César FA, Heras BL, Boscá L, Souza ES, et al. Antiinflammatory and antioxidant properties of a new arylidene-thiazolidinedione in macrophages. Curr Med Chem. 2011;18:3351-60.

33. Lelubre C, Vincent J-L. Mechanisms and treatment of organ failure in sepsis. Nat Rev Nephrol. 2018;14:417-27.

34. Geldenhuys WJ, Nair RR, Piktel D, Martin KH, Gibson LF. The MitoNEET ligand NL-1 mediates antileukemic activity in drug-resistant B-cell acute lymphoblastic leukemia. J Pharm Exp Ther. 2019;370:25-34.

35. Wiley SE, Murphy AN, Ross SA, van der Geer P, Dixon JE. MitoNEET is an ironcontaining outer mitochondrial membrane protein that regulates oxidative capacity. Proc Natl Acad Sci USA 2007;104:5318-23.

36. Yan J, Li S, Li S. The role of the liver in sepsis. Int Rev Immunol. 2014;33:498-510.

37. Robinson MW, Harmon C, O'Farrelly C. Liver immunology and its role in inflammation and homeostasis. Cell Mol Immunol. 2016;13:267-76. 
38. Mantzarlis K, Tsolaki V, Zakynthinos E. Role of oxidative stress and mitochondrial dysfunction in sepsis and potential therapies. Oxid Med Cell Longev. 2017;2017:5985209.

39. Lee S, Nakahira K, Dalli J, Siempos II, Norris PC, Colas RA, et al. NLRP3 inflammasome deficiency protects against microbial sepsis via increased lipoxin B4 synthesis. Am J Respir Crit Care Med. 2017;196:713-26.

40. Geto Z, Molla MD, Challa F, Belay Y, Getahun T. Mitochondrial dynamic dysfunction as a main triggering factor for inflammation associated chronic noncommunicable diseases. J Inflamm Res. 2020;13:97-109.

41. Lee HJ, Choi JS, Lee HJ, Kim W-H, Park SI, Song J. Effect of excess iron on oxidative stress and gluconeogenesis through hepcidin during mitochondrial dysfunction. J Nutr Biochem. 2015;26:1414-23.

42. Reelfs O, Abbate V, Cilibrizzi A, Pook MA, Hider RC, et al. The role of mitochondrial labile iron in Friedreich's ataxia skin fibroblasts sensitivity to ultraviolet A. Metallomics 2019;11:656-65.

43. Furihata T, Takada S, Maekawa S, Mizushima W, Watanabe M, Takahash $\mathrm{H}$, et al. mitoNEET regulates mitochondrial iron homeostasis interacting with transferrin receptor. Circulation. 2017;136:A17534.

44. Habener A, Chowdhury A, Echtermeyer F, Lichtinghagen R, Theilmeier G, Herzog C. MitoNEET protects $\mathrm{HL}-1$ cardiomyocytes from oxidative stress mediated apoptosis in an in vitro model of hypoxia and reoxygenation. PLoS ONE. 2016;11: e0156054.

45. Huynh N, Ou Q, Cox P, Lill R, King-Jones K. Glycogen branching enzyme controls cellular iron homeostasis via iron regulatory protein 1 and mitoNEET. Nat Commun. 2019;10:5463.

46. Zuris JA, Ali SS, Yeh $H$, Nguyen TA, Nechushtai $R$, Paddock $M L$, et al. NADPH Inhibits [2Fe-2S] Cluster Protein Transfer from Diabetes Drug Target MitoNEET to an Apo-acceptor Protein. J Biol Chem. 2012;287:11649-55.

47. Hunter RL, Choi D-Y, Ross SA, Bing G. Protective properties afforded by pioglitazone against intrastriatal LPS in Sprague-Dawley rats. Neurosci Lett. 2008:432:198-201.

48. Hoeft K, Bloch DB, Graw JA, Malhotra R, Ichinose F, Bagchi A. Iron loading exag gerates the inflammatory response to the toll-like receptor 4 ligand lipopolysaccharide by altering mitochondrial homeostasis. Anesthesiology. 2017;127:121-35.

49. Kwon M-Y, Hwang N, Park Y-J, Perrella MA, Chung SW. NOD2 deficiency exacerbates hypoxia-induced pulmonary hypertension and enhances pulmonary vascular smooth muscle cell proliferation. Oncotarget. 2018;9:12671-81.

50. Kwon M-Y, Ghanta S, Ng J, Tsoyi K, Lederer JA, Bronson RT. Expression of stromal cell-derived factor- 1 by mesenchymal stromal cells impacts neutrophil function during sepsis. Crit Care Med. 2021;48:e409-17.

\section{ACKNOWLEDGEMENTS}

This research was supported by the Basic Science Research Program through the National Research Foundation of Korea (NRF) funded by the Ministry of Education and the KIST Institutional Program (NRF-2014R1A6A1030318 and NRF2021R1A2C1094382 to S.W.C; NRF-2019R1A2C1086600 and 2E1390- 21-P017 to S.J. L.).

\section{AUTHOR CONTRIBUTIONS}

Conceptualization, S.W.C., S-J.L.; Methodology, S.L.; Validation, S.L., B.G.S., S-J.L., and S. W.C.; Formal Analysis, S.L., B.G.S.; Investigation, S.L., B.G.S.; Writing Original Draft Preparation, S.W.C., S.L; Writing Review \& Editing, S.W.C., S.L.; Visualization, S.L.; Supervision, S.W.C.; Project Administration, S.W.C.; Funding Acquisition, S.W.C., S-J.L.

\section{COMPETING INTERESTS}

The authors declare no competing interests.

\section{ADDITIONAL INFORMATION}

Supplementary information The online version contains supplementary material available at https://doi.org/10.1038/s41419-022-04586-2.

Correspondence and requests for materials should be addressed to Su Wol Chung.

Reprints and permission information is available at http://www.nature.com/ reprints

Publisher's note Springer Nature remains neutral with regard to jurisdictional claims in published maps and institutional affiliations.

Open Access This article is licensed under a Creative Common C. Attribution 4.0 International License, which permits use, sharing, adaptation, distribution and reproduction in any medium or format, as long as you give appropriate credit to the original author(s) and the source, provide a link to the Creative Commons license, and indicate if changes were made. The images or other third party material in this article are included in the article's Creative Commons license, unless indicated otherwise in a credit line to the material. If material is not included in the article's Creative Commons license and your intended use is not permitted by statutory regulation or exceeds the permitted use, you will need to obtain permission directly from the copyright holder. To view a copy of this license, visit http://creativecommons. org/licenses/by/4.0/.

(c) The Author(s) 2022 